\title{
Effect of alcohol intake on the severity of injuries caused by slipping down
}

\author{
Kyoung Sung Yun ${ }^{1}$, Jin-Seong $\mathrm{Cho}^{2}$, Yong Su Lim², Jae Ho Jang ${ }^{2}$, \\ Hyuk Jun Yang ${ }^{2}$, Woo Sung Choi ${ }^{1}$ \\ 'Department of Emergency Medicine, Gachon University Gil Hospital, Incheon, Korea \\ ${ }^{2}$ Department of Emergency Medicine, Gil Medical Center, Gachon University College of Medicine, Incheon, \\ Korea
}

Objective Alcohol consumption is associated with an increased risk of injuries. However, its effects on injury severity and mortality remain unclear. Specifically, the effects of alcohol intake on the severity of slip injuries have not been thoroughly investigated. Therefore, our study aimed to investigate the effects of alcohol intake on injury patterns and severity in patients experiencing slip injuries.

Methods Emergency department surveillance data collected from 2011 to 2016 were analyzed for this study. Among patients aged 15 and older who were admitted for slip injuries, we compared the type and severity of injuries between the alcohol-intake group and the no-alcohol-intake group. Injury severity was classified as non-severe and severe based on the excess mortality ratio-adjusted injury severity score.

Results In total, 227,548 (alcohol-intake, $n=48,581$; no-alcohol-intake, $n=178,967$ ) patients were included. After adjusting for age, time of injury, use of public ambulance, and season, multivariate logistic regression analysis showed that injuries were more likely to be severe in the alcohol-intake group than in the no-alcohol-intake group (odds ratio, 1.60; 95\% confidence interval, 1.47-1.75). In addition, male gender and alcohol consumption had a greater synergistic effect on injury severity than the mere sum of each effect of these factors (odds ratio, 2.65; 95\% confidence interval, 2.53-2.78).

Conclusion Assessment of the patients influenced by alcohol was a challenge in the emergency department due to altered mental status. We suggest a considerate approach in testing and assessing male patients who slipped after alcohol-intake in the emergency department.

Keywords Alcohol drinking; Accidental falls; Trauma severity indices

Capsule
Wummary is already known
It is known that alcohol consumption is an important risk factor for injuries be-
cause drinking alcohol leads to a decrease in body maneuverability and a de-
crease in the body's ability to withstand injuries.
What is new in the current study
The severity of an injury can be increased in patients who slip after drinking al-
cohol. In addition, male gender and alcohol-intake might show a synergistic ef-
fect greater than the mere sum of their effects on the severity of injuries. Our
study suggests the need for more thorough examinations and careful testing
among male patients drinking alcohol presenting to the emergency department
after slipping down.

Received: 12 July 2019

Revised: 26 August 2019

Accepted: 3 September 2019

Correspondence to: Jin-Seong Cho Department of Emergency Medicine, Gil Medical Center, Gachon University College of Medicine, 783 Namdongdaero, Namdong-gu, Incheon 21556, Korea

E-mail: cjs@gilhospital.com ORCID

https://orcid.org/0000-0001-6762-4692

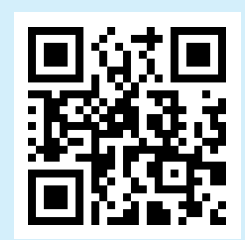

How to cite this article:

Yun KS, Cho JS, Lim YS, Jang JH, Yang HJ, Choi WS. Effect of alcohol intake on the severity of injuries caused by slipping down. Clin Exp Emerg Med 2020;7(3):170-175. https:// doi.org/10.15441/ceem.19.062

This is an Open Access article distributed under the terms of the Creative Commons Attribution Non-Commercial License (https:// creativecommons.org/licenses/by-nc/4.0/). 


\section{INTRODUCTION}

More than 70\% of South Korea's population consumes alcohol, and alcohol-related costs have been increasing annually. As of 2015 , costs were estimated to be 9.45 trillion Korean won. ${ }^{1,2}$ Beyond these costs, alcohol-related injuries present major societal challenges. Drinking leads to a decrease in body maneuverability as well as decreased ability to withstand injuries. Several studies have that alcohol consumption was an important risk factor for injuries, and it has been reported that almost 50\% of trauma patients are injured while under the influence of alcohol. ${ }^{3-7}$

Many studies have examined the effects of alcohol on injury severity and mortality across various groups of injured patients. ${ }^{8-12}$ However, none of these studies focused solely on the mechanism of injuries due to slips. We have thought that patients with slip injuries, who are commonly seen in emergency departments (EDs), tend to be less thoroughly examined than those experiencing traffic accidents or accidents involving a fall. Moreover, patients who experience slips after alcohol intake experience an altered mental state, which makes it difficult to conduct medical history reviews and physical examination. Because the evaluation of injury severity is challenging in these patients, it is difficult to decide the type of examination and treatment required.

Given these challenges, this study aimed to investigate the effects of alcohol intake on injury patterns and severity in patients experiencing slip injuries.

\section{METHODS}

\section{Data collection}

This was a retrospective cohort study, performed using data available in the Emergency Department-based Injury In-depth Surveillance (EDIIS) database, which is collected and managed by the Korea Centers for Disease Prevention and Control. The EDIIS is a national prospective database containing information from injured patients presenting to an ED, in which injury-related data are collected to aid in devising injury-related national policies. Twentythree hospitals nationwide currently supply information to this database. This database was developed based on the International Classification of External Causes of Injuries by the World Health Organization. The database contains information about patients' demographic characteristics, injury, emergency care records, ED diagnosis, ED treatment, discharge, and hospital admission, and patient outcomes after hospital admission. The data are collected by ED physicians and/or emergency medical technicians, and the collected data are supenised and corrected by a skilled research coordinator in charge of the EDIIS. The research coordinator, who completed a relevant education program prior to participating in the project, inputs the collected data into the web-based database system. The Quality Control Committee reviews the database and periodically provides feedback to the EDIIS participants.

\section{Participants}

We utilized EDIIS data from January 1, 2011 to December 31, 2016. The study participants were patients aged 15 years or older who presented to the ED of one of the study hospitals for an injury after slipping, and not a fall-down injury. Patients suspected of visiting the ED after visiting a previous hospital and not immediately visiting the ED after injury were excluded from the study. Patients conducting self-harm/violence were also excluded to allow for the selection of patients in whom the slip injuries were not intentional. Patients lacking data important for the study, such as injury severity, were also excluded.

\section{Definition of variables}

Patients' sex, age, time of injury, methods of hospital transport, and other information were collected. We confirmed sex and age distribution in relation to alcohol intake. Time of injury was classified into day ( $\geq 08: 00,<16: 00)$, evening ( $\geq 16: 00,<24: 00)$, and night ( $\geq 00: 00,<08: 00$ ). Methods of hospital transport were classified into the use of a public ambulance and non-use of public ambulance. Next, we confirmed how specific seasons and locations affected the occurrence of slip injuries in relation to alcohol intake. To determine whether there were differences in the anatomic region affected between the alcohol-intake and no-alcohol-intake groups, the frequency of injuries to the head and neck, chest, abdomen, and pelvis, and extremities was documented.

Injury severity was classified as non-severe and severe based on the excess mortality ratio-adjusted injury severity score (EMR-ISS). The EMR-ISS is a system for assessing injury severities based on the International Statistical Classification of Diseases and Related Health Problems 10th revision (ICD-10). With this method, the excess mortality ratio is computed for all ICD-10 codes in the Korean National Injury Database to classify the ICD-10 codes into grades 1 to 5 , and the EMR-ISS is calculated by summing the squares of the three highest severity scores. ${ }^{13-15}$ Based on the total EMR-ISS score, injuries are classified into mild (1-8), moderate (9-24), severe (25-74), and critical ( $\geq 75$ ). Patients with an EMR-ISS classification of mild and moderate are usually included in the non-severe group, while those with an EMR-ISS classification of severe and critical are included in the severe group..$^{16,17}$

Finally, in our study, patient prognosis was assessed based on the outcomes of ED treatment (discharge, transfer, admission, or death) and mortality. 


\section{Statistical analysis}

The differences in characteristics between the alcohol-intake and no-alcohol-intake groups were assessed using chi-square tests and the Mann-Whitney U-test. To identify the risk factors for severe injuries, covariates with a P-value $<0.1$ in the univariate analysis and clinically important covariates were entered into a multivariate logistic regression with backward stepwise elimination. Data were analyzed using the Stata ver. 13.1 (StataCorp., College Station, TX, USA). When sex and alcohol-intake factors were involved, analysis for identifying the interaction effects between these factors was performed. The assessment was conducted by calculating relative excess risk caused by interaction (RERI) using the Anderson algorithm. RERI was obtained by subtracting the values of the odds ratio for female sex and alcohol intake and the odds ratio for male sex and no alcohol intake from the value of the odds ratio for the simultaneous presence of both risk factors and adding 1.

\section{Ethics statement}

The present study was reviewed and approved by the institutional review board of Gachon University Gil Medical Center (GCIRB2016242). The institutional review board waived the requirement for obtaining a signed informed consent because it was a retrospective study where personal information could not be verified.

\section{RESULTS}

In total, 365,979 patients were examined; following the application of exclusion criteria, 227,548 patients were included in the

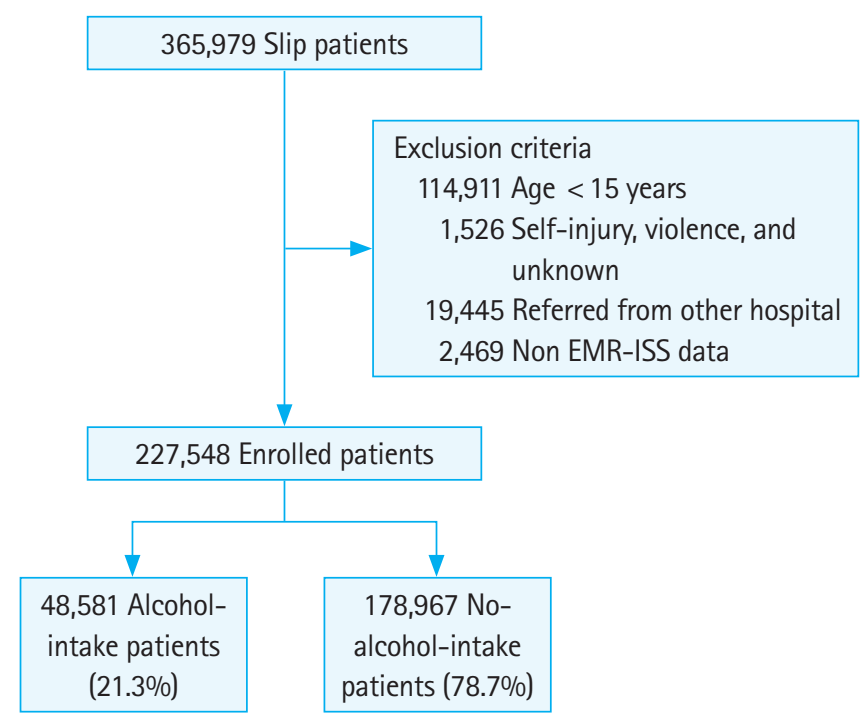

Fig. 1. Flow chart of patient selection. EMR-ISS, excess mortality ratioadjusted injury severity score. final analysis. Of the included patients, 48,581 (21.3\%) and 178,967 (78.7\%) were classified into the alcohol-intake group and no-alcohol-intake group, respectively (Fig. 1).

The alcohol-intake group was younger and had a higher proportion of males $(P<0.001)$ than the no-alcohol-intake group. $A$

Table 1. Baseline characteristics of patients experiencing slips

\begin{tabular}{|c|c|c|c|}
\hline Variable & No-alcohol-intake & Alcohol-intake & P-value \\
\hline Total & 178,967 & 48,581 & \\
\hline Age (yr) & $54.0(34.0-71.0)$ & $44.0(31.0-55.0)$ & $<0.001$ \\
\hline Sex, male & $78,914(44.1)$ & $36,105(74.3)$ & $<0.001$ \\
\hline Time of injury (hr) & & & $<0.001$ \\
\hline$\geq 00: 00,<08: 00$ & $38,961(21.8)$ & $22,249(45.8)$ & \\
\hline$\geq 08: 00,<16: 00$ & $70,266(39.3)$ & $3,699(7.6)$ & \\
\hline$\geq 16: 00,<24: 00$ & $68,438(38.2)$ & $22,316(45.9)$ & \\
\hline Injury circumstance & & & $<0.001$ \\
\hline Work & $33,775(18.9)$ & $8,556(17.6)$ & \\
\hline Activities of daily living & $139,335(77.9)$ & $38,270(78.8)$ & \\
\hline Miscellaneous (travel, other) & $5,389(3.3)$ & $1,755(3.6)$ & \\
\hline \multicolumn{3}{|c|}{ Transportation to the emergency department } & $<0.001$ \\
\hline Public ambulance & $47,506(26.5)$ & $21,664(44.6)$ & \\
\hline Season & & & $<0.001$ \\
\hline Spring & $41,488(23.2)$ & $12,490(25.7)$ & \\
\hline Summer & $41,144(23.0)$ & $10,289(21.2)$ & \\
\hline Autumn & $44,131(24.7)$ & $12,077(24.9)$ & \\
\hline Winter & $52,166(29.1)$ & $13,687(28.2)$ & \\
\hline Location & & & $<0.001$ \\
\hline Indoor & $83,915(46.9)$ & $16,562(34.1)$ & \\
\hline Outdoor & $93,596(52.3)$ & $31,561(65.0)$ & \\
\hline Unknown & $1,456(0.8)$ & $458(0.9)$ & \\
\hline
\end{tabular}

Values are presented as number, median (interquartile range), or number (\%).

Table 2. Injury characteristics of patients experiencing slips

\begin{tabular}{lccc}
\hline Characteristics & No-alcohol-intake & Alcohol-intake & P-value \\
\hline Total & 178,967 & 48,581 & \\
EMR-ISS & & & $<0.001$ \\
$\quad$ Non-severe $(1 \leq,<25)$ & $168,190(94.0)$ & $44,034(90.6)$ & \\
$\quad$ Moderate $(25 \leq,<75)$ & $10,777(6.0)$ & $4,547(9.4)$ & \\
Affected area & & & \\
Head and neck & $76,910(42.9)$ & $37,398(77.0)$ & $<0.001$ \\
Chest & $9,093(5.1)$ & $1,309(2.7)$ & $<0.001$ \\
Abdomen and pelvis & $12,035(6.7)$ & $940(1.9)$ & $<0.001$ \\
Extremities & $82,728(46.2)$ & $8,675(17.9)$ & $<0.001$ \\
ED outcome & & & $<0.001$ \\
Discharge & $141,455(79.0)$ & $43,040(88.6)$ & \\
Transfer & $5,690(3.2)$ & $1,063(2.2)$ & \\
Admission & $31,475(17.6)$ & $4,156(8.6)$ & \\
Death in the ED & $109(0.7)$ & $29(0.7)$ & \\
Total no. of deaths & $878(0.5)$ & $142(0.3)$ & $<0.001$ \\
\hline
\end{tabular}

Values are presented as number or number (\%).

EMR-ISS, excess mortality ratio-adjusted injury severity score; ED, emergency department. 
Table 3. Effect of the interaction between alcohol intake and sex on the risk of severe injury

\begin{tabular}{lcccccc}
\hline & \multicolumn{2}{c}{ No-alcohol-intake } & & \multicolumn{2}{c}{ Alcohol-intake } & \multicolumn{2}{c}{$\mathrm{OR}^{\text {a) }}(95 \% \mathrm{Cl})$ for alcohol } \\
intake based on sex
\end{tabular}

Measure of interaction on an additive scale $(95 \% \mathrm{Cl})=0.25(0.09-0.41)$; Measure of interaction on a multiplicative scale $(95 \% \mathrm{Cl})=0.92(0.83-1.01)$.

$\mathrm{OR}$, odds ratio; $\mathrm{Cl}$, confidence interval.

${ }^{a}$ ORs are adjusted for age, injury time, use of ambulance, and season.

significantly higher proportion of injuries in the alcohol-intake occurred during the evening ( $\geq 16: 00,<24: 00)$ and night ( $\geq 00: 00$, $<08: 00)$ than during the daytime $(\geq 08: 00,<16: 00)(P<0.001)$. The rate of public ambulance usage and proportion of injuries sustained outdoors was also higher in the alcohol-intake group than in the no-alcohol-intake group (Table 1).

When the severity of injuries was classified according to the EMR-ISS, the proportion of patients with severe injuries in the no-alcohol-intake and alcohol-intake groups was $6.0 \%$ and $9.4 \%$, respectively $(\mathrm{P}<0.001)$ (Table 2$)$. The alcohol-intake group had a lower frequency of injuries to the extremities than the no-alcohol-intake group, but a higher frequency of head injuries $(P<0.001)$ (Table 2).

After adjusting for age, time of injury, use of a public ambulance, and season, multivariate logistic regression analysis showed that injuries were more likely to be severe in the alcohol-intake group than in the no-alcohol-intake group (odds ratio [OR], 1.60; 95\% confidence interval [CI], 1.47-1.75) (Table 3). RERI assessment demonstrated interaction effects between male sex and alcohol intake (RERI, 0.25; 95\% Cl, 0.09-0.41). Therefore, male gender and alcohol intake had an additive interaction effect that was greater than the sum of the independent effects of these factors on injury severity $(\mathrm{OR}, 2.65 ; 95 \% \mathrm{Cl}, 2.53-2.78)$.

\section{DISCUSSION}

In our study of patients experiencing slips, the alcohol-intake group experienced more severe injuries than the no-alcohol-intake group $(\mathrm{OR}, 1.60 ; 95 \% \mathrm{Cl}, 1.47-1.75)$. A prior study that utilized ordinal logistic regression with an EMR-ISS-based five-group classification also showed that the alcohol-intake group was more likely to experience severe injuries, although the effect reported in this previous study was much stronger $(\mathrm{OR}, 2.27 ; 95 \% \mathrm{Cl}, 1.40-3.68)$. However, this study focused on bicycle-related injuries only and included only 689 patients. ${ }^{7}$ Another study reported more severe injuries and higher mortality rates in trauma patients who tested positive for alcohol in the blood. However, this study included all patients who required hospitalization owing to various traumas, the number of patients in the study was relatively small (361 patients), and multivariate analysis was not performed. ${ }^{9}$

One study involving patients admitted to multiple trauma centers reported that there were no significant differences in mortality between those with blood alcohol concentrations of $<0.1 \%$ and $\geq 0.1 \%$. However, this study only focused on deaths and not the mechanism or severity of injury. ${ }^{10}$ Another study also reported no significant difference between injuries due to road traffic accidents, crashes, and falls between patients who had consumed alcohol and those who had not. ${ }^{12}$ However, this study focused only on patients at a local emergency medical center and the results reflected this specific setting.

In contrast, the present study used a unique and novel approach by examining the effect of alcohol intake on the severity of injuries due to slipping down and found a correlation between alcohol intake and increased injury severity. The results confirm that alcohol intake is associated with an increased risk of injury, and in the case of slips, alcohol may also lead to more severe injuries. This information suggests the need for more comprehensive testing and treatment for alcohol-related injuries due to slipping.

In our study, we found that there was significantly higher percentage of males in the alcohol-intake group than in the no-alcohol-intake group, consistent with data from prior studies showing that alcohol-induced injuries are more common in males than in females. ${ }^{9,11,12,18,19}$ In the case of slips, our study showed that males were more likely to experience severe injuries than females (OR, 1.80; 95\% Cl, 1.72-1.87). Additional interaction effects between male sex and alcohol intake on injury severity were also observed (OR, 2.65; 95\% Cl, 2.53-2.78). This may be because even among those who consumed alcohol, male patients showed more patterns of heavy drinking than female. Given these results, more active examinations and treatments should be considered for male drinkers, with a focus on visits to the ED after injuries from slipping.

The alcohol-intake group was more likely to visit to the ED in the early morning ( $\geq 00: 00,<08: 00)$, reflecting that early-morn- 
ing drinking is more common than drinking during other times. Another study on traumatic brain injury reported that patients who had consumed alcohol were more likely to report in the night (00:00-08:00) (52.1\%) than the no-alcohol-intake group (24.8\%). The proportion of patients who visited the clinic from 20:00 to 8:00 on the next day for the examination of blunt-force injuries was $77.7 \%$ among the alcohol-intake group, and $62 \%$ in the noalcohol-intake group. ${ }^{11,12}$ While we did not examine the geographic locations where injuries occurred in our study, we hypothesize that if a patient who has consumed alcohol slips, this slip is likely to occur in a dark place, and patients are more likely to be discovered late in the night-time than at other times.

Hundreds of diagnostic ICD-10 codes currently exist, making it difficult to individually identify the distribution of a particular code. Thus, we categorized injury codes based on four anatomical areas and examined the related frequency. The alcohol-intake group had a lower frequency of injury to the extremities than the no-alcohol-intake group, but exhibited a higher frequency of head injuries, increasing the severity of alcohol-intake-related injuries.

In our study, the alcohol-intake group showed higher injury severity, higher discharge rate, and lower admission and transfer rates than the no-alcohol-intake group. These results can be explained by the correlation between alcohol intake and socioeconomic status. A study of 8,000 adult men and women reported that lower educational levels increased the prevalence of alcoholrelated problems by three- or four-fold, and another study found that alcohol abuse or dependence increased a few years after job loss. Taken in the context of these findings, our results suggest that patients experiencing slips in the alcohol-intake group were socioeconomically more vulnerable than those in the no-alcoholintake group. This could lead to a drop in their ED admission rate, irrespective of injury severity. ${ }^{20,21}$

Although our study was performed across several hospital sites and indications, it is not without limitations. First, this study could not include comorbidities as a variable in the multivariate analysis because this information could only be collected from 7 of the 23 hospitals (resulting in a 60\%-70\% miss rate). Second, the present study classified a patient into the alcohol-intake group when the patient admitted to drinking or when the examiner suspected alcohol intake after a physical examination. However, blood alcohol concentration was not measured. Due to a lack of appropriate equipment, legal, and ethical limitations, assessment of blood alcohol concentration was difficult in many cases. Moreover, the self-report method of alcohol intake has frequently been used in other injury studies and has shown high sensitivity and specificity comparable to that of the measurement of blood alcohol concentration. ${ }^{22,23}$ Therefore, the reliability of the results of the present study is considered acceptable. Third, the EMR-ISS is based on a patient's diagnosis, calculates the excess mortality ratio of the patient based on the diagnosis, and indicates the trauma severity for each diagnosis. The values are calculated based on the diagnosis recorded on the acquired data and may lead to lowered objectivity, possibly contributing to the differences in prognosis noted in comparison with other studies using EMR-ISS.

In summary, in this study using ED-based data on patients experiencing slips, we found that injury severity was higher among those consuming alcohol. Considering the difficulty in ED assessments for patients who have consumed alcohol, the results of the present study underline the need for more thorough examinations and careful testing, especially among male patients experiencing slips after alcohol intake.

\section{CONFLICT OF INTEREST}

No potential conflict of interest relevant to this article was reported.

\section{ACKNOWLEDGMENTS}

The authors are grateful to the Korea Center for Disease Control and Prevention for supporting the EDIIS database.

\section{REFERENCES}

1. Ministry of Health and Welfare. National Health Nutrition Survey: trend of annual drinking rate 2005-2017 [Internet]. Deajeon: Korean Statistical Information Service; 2019 [cited 2019 Feb 20]. Available from: http://kosis.kr/statHtml/statHtml.do?orgld = 117\&ttblld = DT_11702_N011\&conn_path = I2 19.

2. National Health Insurance Corporation. Impact of socioeconomy and evaluation of the effectiveness of regulatory policy of major health problem [Internet]. Wonju: National Health Insurance Corporation; 2015 [cited 2019 Feb 20]. Available from: https://www.nhis.or.kr/menu/boardRetriveMenuSet. xx?menuld $=$ F3430

3. D'Onofrio G, Bernstein E, Bernstein J, et al. Patients with alcohol problems in the emergency department, part 1: improving detection. SAEM Substance Abuse Task Force. Society for Academic Emergency Medicine. Acad Emerg Med 1998;5:1200-9.

4. Dunn CW, Donovan DM, Gentilello LM. Practical guidelines for performing alcohol interventions in trauma centers. J Trauma 1997:42:299-304.

5. Xiang $X$, Luo $T$, Wang $X$, Hao W. Relationship between injury 
and alcohol use in 531 patients in the emergency room. Zhong Nan Da Xue Xue Bao Yi Xue Ban 2014;39:723-6.

6. Borges $G$, Cherpitel C, Mittleman M. Risk of injury after alcohol consumption: a case-crossover study in the emergency department. Soc Sci Med 2004;58:1191-200.

7. Sethi $M$, Heyer JH, Wall $S$, et al. Alcohol use by urban bicyclists is associated with more severe injury, greater hospital resource use, and higher mortality. Alcohol 2016;53:1-7.

8. Kim HB, Lee $K H$, Kim H, et al. Risk factors affecting severity of injury in patients of motor cycle accidents. J Korean Soc Emerg Med 2011;22:226-30.

9. Choi YH, Kim GB, Lee DH, et al. Effect of alcohol ingestion on the severity and outcome in trauma patients. J Korean Soc Emerg Med 2006;17:231-7.

10. Zeckey C, Dannecker S, Hildebrand F, et al. Alcohol and multiple trauma: is there an influence on the outcome? Alcohol 2011;45:245-51.

11. Cho JS, Shin SD, Lee EJ, et al. Alcohol intake and reduced mortality in patients with traumatic brain injury. Alcohol Clin Exp Res 2016;40:1290-4.

12. Lee EJ, Kim TY, Kim SC, et al. Effect of alcohol consumption on the severity of blunt injury. J Korean Soc Emerg Med 2005; 16:339-45.

13. Kim J, Shin SD, Im TH, et al. Development and validation of the excess mortality ratio-adjusted Injury Severity Score using the International Classification of Diseases 10th edition. Acad Emerg Med 2009;16:454-64.

14. Cha MI, Kim GW, Kim CH, et al. A study on the disaster medical response during the Mauna Ocean Resort gymnasium col- lapse. Clin Exp Emerg Med 2016;3:165-74.

15. Park JH, Park JO, Ro YS, Shin SD. Effect of alcohol use on emergency department length of stay among minimally injured patients based on mechanism of injury: multicenter observational study. Clin Exp Emerg Med 2018;5:7-13.

16. Choi JY, Cho JS, Lim YS, et al. Effect of age on severity of injuries in traffic accidents involving elderly drivers. Int J Gerontol 2019;13:246-50.

17. Lee HH, Cho JS, Lim YS, et al. Relationship between age and injury severity in traffic accidents involving elderly pedestrians. Clin Exp Emerg Med 2019;6:235-41.

18. Demetriades D, Gkiokas G, Velmahos GC, Brown C, Murray J, Noguchi T. Alcohol and illicit drugs in traumatic deaths: prevalence and association with type and severity of injuries. J Am Coll Surg 2004;199:687-92.

19. Lee WK, Kim Y. Drinking pattern and nonfatal injuries of adults in Korea. J Korean Soc Traumatol 2009;22:233-41.

20. Van Oers JA, Bongers IM, van de Goor LA, Garretsen HF. Alcohol consumption, alcohol-related problems, problem drinking, and socioeconomic status. Alcohol Alcohol 1999;34:78-88.

21. Collins SE. Associations between socioeconomic factors and alcohol outcomes. Alcohol Res 2016;38:83-94.

22. Noh $H$, Choi HY, Eo EK, Jung KY. The adequacy of self-reporting and physician's diagnoses for assessing alcohol ingestion in trauma patients. J Korean Soc Emerg Med 2007;18:307-12.

23. Raj $R$, Mikkonen ED, Siironen J et al. Alcohol and mortality after moderate to severe traumatic brain injury: a meta-analysis of observational studies. J Neurosurg 2016;124:1684-92. 